\title{
Hybrid P-Element and Trefftz Method for Capacitance Computation
}

\author{
Miklos Gyimesi, Senior Member IEEE, Jian-She Wang, Member IEEE \\ Dale Ostergaard, Member IEEE and ASME \\ ANSYS, Inc. Southpointe, 275 Technology Dr., Canonsburg, PA 15317, USA

\section{NEAR FIELD BY ADAPTIVE P-ELEMENTS}

\begin{abstract}
The paper discusses efficient electrostatic field solutions techniques to simulate micro electro mechanical systems (MEMS): adaptive p-elements to remove the burden of manual mesh refinement; hybrid finite elements - Trefftz formulation to treat open boundary problems; energy based capacitance matrix and lumped capacitance value extraction.
\end{abstract}

Index terms - Micro Electro Mechanical Systems (MEMS), Finite elements (FE), Trefftz, p-elements, Capacitance, Hybrid FE-Trefftz

\section{INTRODUCTION}

There has been an ever-increasing need for simulation tools to perform virtual prototyping of Micro Electro Mechanical Systems (MEMS). Many MEMS devices apply comb drives to actuate resonators, micromirrors, and switches by electrostatic forces or measure the capacitance change due to beam or membrane deflections in accelerometers, gyroscopes or pressure sensors.

The ANSYS finite element (FE) package provides a wide range of tools to model MEMS devices [1-3]. This paper focuses on ANSYS's Release 5.6 electrostatic enhancements:

- Adaptive p-elements for automatic mesh refinement - Hybrid FE-Trefftz for open boundary treatment

- Capacitance extraction by energy principles

The simulation of MEMS devices requires the accurate characterization of both the near and far electrostatic field around the electrodes. The near-field precision is vital to accurately compute electrostatic forces on structural members. Both the near-field and the far-field play an important role in precise capacitance computations. In addition, multiple dielectric material modeling is necessary to simulate oxide layers, substrates as well as other dielectrics in the device.
ANSYS has traditional electrostatic $h$-elements, where the user controls mesh refinement to increase accuracy by decreasing the characteristic mesh size, $h$. New electrostatic $p$-elements have been introduced in the program which converge the solution by selectively increasing the polynomial order, $p$ of elements. The basic procedure is explained below (see [4] for additional details).

There are basically two aspects in the p-element approach: error assessment and selective $p$-refinement. The core part is the error estimation. Suppose a FE (primary) electric field, $\mathbf{E}$ solution has been computed for an electrostatic problem. A second solution of the displacement vector, $\hat{\mathbf{D}}$, satisfying the Maxwell's equations and boundary conditions, may be constructed $a$ posteriori. Here a solution is sought in a finite element, of the form:

$\hat{\mathbf{D}}={ }_{n} d_{n} \mathbf{F}_{n}$,

where $\mathbf{F}_{n}$ 's are normally continuous, vector functions that belong to the functional space $\mathrm{H}(d i v)$. This specific choice helps to automatically maintain normal continuity of $\mathbf{D}$ on material interfaces. From the pair $(\mathbf{E}, \hat{\mathbf{D}})$, it is now possible to compute the relative constitutive error:

$\varepsilon_{\Omega}=\frac{\|\hat{\mathbf{D}}-[\varepsilon] ? \mathbf{E}\|_{\Omega}}{\|\hat{\mathbf{D}}+[\varepsilon] ? \mathbf{E}\|_{\Omega}}$,

where $[\varepsilon]$ is the permittivity tensor. The global relative error (2) is seen as the sum of element contributions:

$$
e_{\Omega}^{2}=e_{E}^{2},
$$

where the relative error for an element $E$ is given by: 


$$
e_{E}=\frac{\|\hat{\mathbf{D}}-[\varepsilon] ? \mathbf{E}\|_{E}}{\|\hat{\mathbf{D}}+[\varepsilon] ? \mathbf{E}\|_{\Omega}}
$$

The global error (2) helps to quantify the quality of the approximate pair (E, $\hat{\mathbf{D}})$, while the element error (4) allows to localize the error distribution in the solution domain as required for $p$-enrichment.

From a practical point of view, it is preferable to invoke error control based on energy, field, or force quantities. In ANSYS, the following adaptive strategy is employed:

i) Start with a finite element mesh with corner- and mid-nodes (i.e., $p=2$ )

ii) Obtain solution. Iterative equation solver is preferred

iii) Calculate global-wise energy, force, and/or pointwise electric field values. Up to ten such specifications may be requested

iv) Check convergence. Stop if tolerance is met

v) Exit if maximum $p$-level $(=8)$ is reached

vi) Compute element relative error distribution

vii) Raise $p$-level of elements with larger relative constitutive error and go to step ii)

Step (v) is based on the consideration that convergence may not be reached if the starting mesh is too coarse. Under this case, a refined mesh should be generated, and the process may be tried again.

\section{FAR-FIELD BY HYBRID FE-TREFFTZ}

There has been considerable controversy over the years as to whether FE or boundary elements (BE) are better suited to solving field problems. BE is better for farfield open problems with simple geometry and homogeneous medium. FE is better suited to near-field closed problems with complex geometry and inhomogeneous material. A new hybrid FE-Trefftz method has been developed to combine the best of both FE and BE [5]. Trefftz is a BE formulation based on energy principles resulting in FE like elements. The hybrid FE-Trefftz combines the efficiency and sparse nature of FE for modeling the near-field with Trefftz BE formulation with a small (20-100 unknowns) dense matrix to effectively model the far-field.

The hybrid FE-Trefftz method is described in details in [5]; here it is summarized briefly. FE-Trefftz follows the generalized FE formulation [6] to obtain symmetric
FE-like stiffness matrices from energy principles. The non-singular Trefftz-complete function set is derived from the potentials of multipoles according to quasi FE [7]. The representing multipoles are located in the FE region; their number is independent of the FE mesh. The continuity of the potential between FE and Trefftz regions is satisfied according to transfinite elements [8].

The governing equations are:

$\operatorname{div}\left(\varepsilon_{1} \operatorname{grad} U_{1}\right)=g_{1} \quad$ in closed FE domain

$\operatorname{div}\left(\varepsilon_{2} \operatorname{grad} U_{2}\right)=g_{2} \quad$ in open Trefftz domain

where $\varepsilon$ is the permittivity, $U$ is the potential, $g$ is the source, finally subscripts, 1 and 2 denote the FE and Trefftz domains, respectively. Source terms in the Trefftz domain can take into account sources far from the FE region. The Trefftz domain may be inhomogeneous allowing analysis, for example, in a stratified medium. The potential is sought as:

$$
\begin{array}{ll}
U_{1}=\Sigma c_{i} N_{i} & \text { in closed FE domain } \\
U_{2}=\Sigma d_{i} f_{i} & \text { in open Trefftz domain }
\end{array}
$$

where $N_{i}$ are the nodal shape functions in FE domain, $c_{i}$ are the nodal potentials, $d_{i}$ denote Trefftz representing coefficients, i.e. the intensity of multipoles [5] and $f_{i}$ are members of a Trefftz complete function set [6] satisfying a priory:

$\operatorname{div}\left(\varepsilon_{2} \operatorname{grad} f_{i}\right)=0 \quad$ in open Trefftz domain

This selection of the shape functions constitutes the 1 st difference between FE and Trefftz.

The energy of the whole system can be expressed as:

$W=W_{1}+W_{2}$

$W_{1}=1 / 2 \mathbf{c}_{1}^{+} \mathbf{K}_{1} \mathbf{c}_{1}-\mathbf{c}_{1}^{+} \mathbf{g}_{1} \quad$ energy in FE domain

$W_{2}=1 / 2 \mathbf{d}_{2}^{+} \mathbf{K}_{2} \mathbf{d}_{2}-\mathbf{d}_{2}^{+} \mathbf{g}_{2}$ energy in Trefftz domain (12)

where $\mathbf{K}_{1}$ and $\mathbf{K}_{2}$ are the stiffness matrices, $\mathbf{g}_{1}$ and $\mathbf{g}_{2}$ are the load vectors and $\mathbf{c}_{1}$ and $\mathbf{d}_{2}$ are the DOFs of the FE and Trefftz domains, respectively. These can be computed as: 


$$
\begin{array}{ll}
K_{1 i j}=\varepsilon_{1} \operatorname{grad} N_{i} \operatorname{grad} N_{j} d V & \text { FE stiffness } \\
K_{2 i j}=\varepsilon_{2} \operatorname{grad} N_{i} \operatorname{grad} N_{j} d V & \text { Trefftz stiffness } \\
g_{1 i}=N_{i} g_{1} d V & \text { FE load } \\
g_{2 i}=N_{i} g_{2} d V & \text { Trefftz load }
\end{array}
$$

The 2nd difference is that the continuity of the potential is achieved by weighting the residual with functions, $\lambda_{i}[5,8]$ :

$$
\lambda_{i}\left(U_{1}-U_{2}\right) d S=0
$$

This leads to the following constraints:

$$
\mathbf{Q}_{1} \mathbf{c}_{1}+\mathbf{Q}_{2} \mathbf{d}_{2}=0
$$

$$
Q_{1 i j}=\lambda_{i} N_{j} d S
$$

FE constraints

$$
Q_{1 i j}=-\quad \lambda_{i} f_{j} d S
$$

Trefftz constraints

The constrained variation of the energy provides the satisfaction of the governing equations and the continuity of the normal derivative as a natural boundary condition.

The 3rd difference is that the volume integrals of the Trefftz stiffness matrix can be reduced to surface integrals using identities of vector analysis and the Gauss theorem:

$$
K_{2 i j}=f_{i} \varepsilon_{2} \frac{d f_{j}}{d n} d S \quad \text { Trefftz stiffness }
$$

This puts the Trefftz method into the group of boundary formulations albeit the shape functions are defined in the space $[5,6]$.

\section{MULTIPORT CAPACITANCE EXTRACTION}

The self and mutual capacitance values of a multiconductor system are essential in characterization of devices. A designer is interested in lumped capacitance values for use in a circuit-based simulator. A multi-port capacitance matrix extraction tool has been developed for users to conveniently extract lumped capacitance values from the capacitance matrix obtained from field solutions by energy principles.
Consider an arrangement consisting of a couple of electrodes above a ground. This constitutes a multi-port system considering the terminals of an electrode and the ground a port of the system. Denote the vector of potentials of the electrodes with respect to ground by $\mathbf{u}$. Denote the vector of charges of the electrodes by q. The sum of charges including the ground is zero. The relationship between the vector of charges and voltages defines the capacitance matrix

$\mathbf{q}=\mathbf{C} \mathbf{u}$

The charge over the electrodes can be evaluated by a surface integral of the displacement vector. The electrostatic energy can be expressed as

$W=1 / 2 \mathbf{u}^{+} \mathbf{C} \mathbf{u}$

By careful loading of voltages over the electrodes both formulae could serve, in principle, to evaluate the capacitance matrix. In practice, however, the energy method is much more accurate. As a thumb rule, to obtain the same accuracy with the surface integral method as obtained by an energy method, the FE mesh size should be about half resulting about 8 times as many elements. Hieke [10] efficiently obtained accurate capacitance values of MEMS devices with ANSYS's energy based method.

The hybrid FE-Trefftz method is especially suitable to provide accurate energy values because the FE method is energy based and the far field as well as its energy are properly represented by the Trefftz method. Compared to FE with infinite elements, the hybrid FE-Trefftz method provides about an order of magnitude better accuracy (see Fig. 5.).

After the capacitance matrix is evaluated, it can be equivalently replaced by lumped capacitors [9] which can be treated by SPICE like circuit simulators. ANSYS is equipped with a capacitance matrix extraction tool which conveniently provides the capacitances of the equivalent lumped capacitors of a multi-port electrode system [1,2].

\section{ILLUSTRATIVE EXAMPLES}

Figure 1 shows the potential distribution around a comb finger of a MEMS device. The stationary and moving electrodes are set to different potentials. The steepest change in the potential distribution occurs between the electrodes where most of the energy is stored. 
Figure 2 illustrates the $p$-level to obtain a forceconverged solution. In most of the highest energy density region between the electrodes, $p=3$ proved to be enough since the E field is homogeneous; an even hexahedron mesh would be fine here. The highest $p$-levels $(p=7)$ are required near the edges of the electrodes where the field changes rapidly; here an uneven tetrahedron mesh would be the best. Fortunately, the adaptive $p$-method removes the burden from the user to create a finely tuned mesh.

Figure 3 depicts the FE mesh around a spherical electrode of a given potential with respect to the other electrode at infinity. A problem with analytic solution has been chosen so as to monitor the accuracy of the solution. Since the domain is open, a traditional FE approach would require a mesh truncation far enough from the sphere. The FE region is truncated by a rectangular block.

To model the field properly beyond to block, the hybrid FE-Trefftz method is applied with a Trefftz domain exterior to the block. The equipotential surfaces around the sphere are plotted on Figure 4. No mesh truncation effects can be observed thanks to the hybrid FE-Trefftz method.

Traditional FE can ease somewhat on the truncation problem by loading the truncating surface of the rectangular block with infinite elements. It can be seen on Figure 5 that the FE-Trefftz method provides much more accurate capacitance than the infinite elements.

\section{REFERENCES}

[1] D.Ostergaard, "Tooling up for MEMS", ANSYS Solutions, Vol. 1. No.3, Winter, 1999.

[2] M.Gyimesi, D.Ostergaard, "Electro Mechanical Transducer for MEMS in ANSYS", MSM 1999.

[3] D.Ostergaard, M.Gyimesi, "FE Based Reduced Order Modeling of MEMS", MSM 2000.

[4] J-S.Wang, N.Ida, "Three-Dimensional $p$ Adaptive Computation of Electrostatic Force and Energy", COMPUMAG 99, Sapporo, Japan.

[5] M.Gyimesi, D.Lavers, T.Pawlak, D.Ostergaard, "FE-Trefftz", IEEE Trans. MAG Vol.32. pp.671-4, 1996.

[6] A.P.Zielinski, O.C.Zienkiewitz: "Generalized FE with T-Sets," I.J.N.M.E., Vol.21, pp.509$528,1985$.

[7] I.D.Mayergoyz, M.V.C.Chari, A.Konrad: "Boundary Method for 3D FEM." IEEE Trans. MAG. Vol.19, No.6, pp.2333,1983.

[8] J.F.Lee, Z.J.Cendes: "Transfinite Elements", J.Appl.Phys.Vol.61. No.8, pp.3913-15, April 1987.
[9] I.Vago, M.Gyimesi, Electromagnetic Fields, Academic Press, ISBN 963057 4535, Budapest, 1998.

[10] Andreas Hieke, "ANSYS APDL for capacitance computation”, MSM 1999. April 1999, Puerto Rico
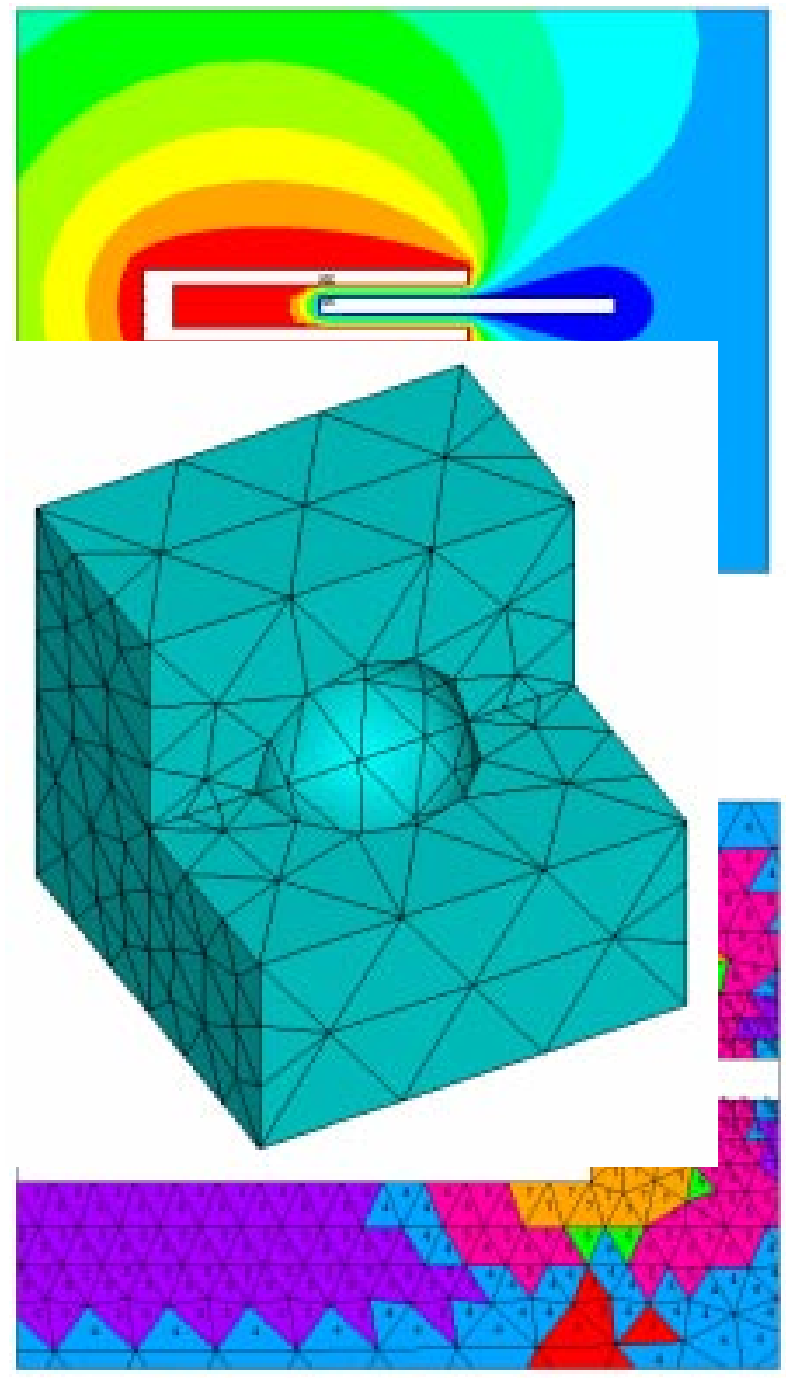

Figure 2: Adaptive p-level Around Comb Finger 


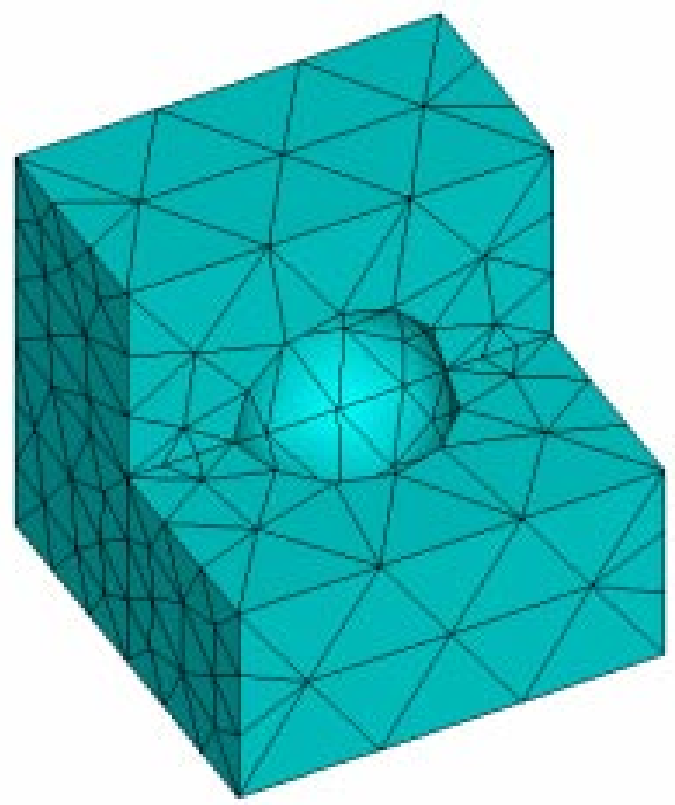

Figure 3: FE model of sphere and surrounding air. Trefftz region is exterior to the block

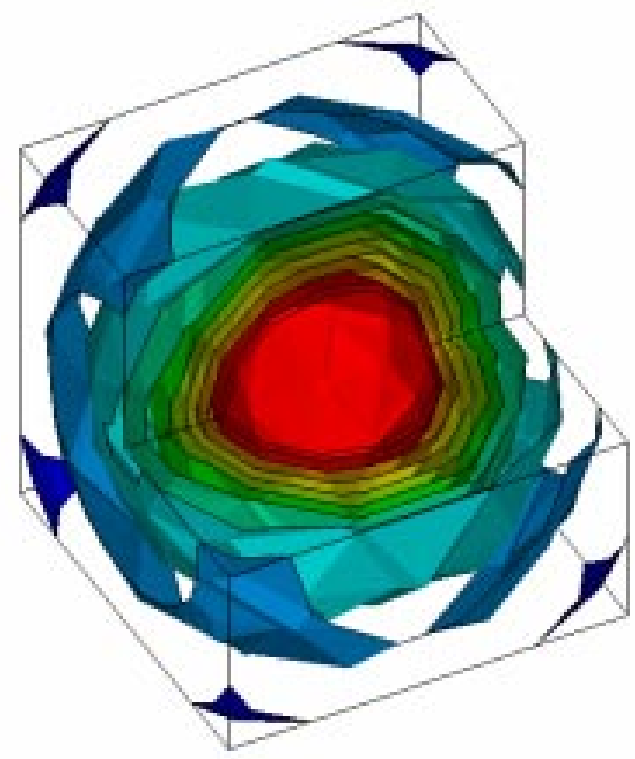

Figure 4: Equipotential distribution around sphere. Note no truncation behavior at FE boundary

\section{Capacitance Convergence}

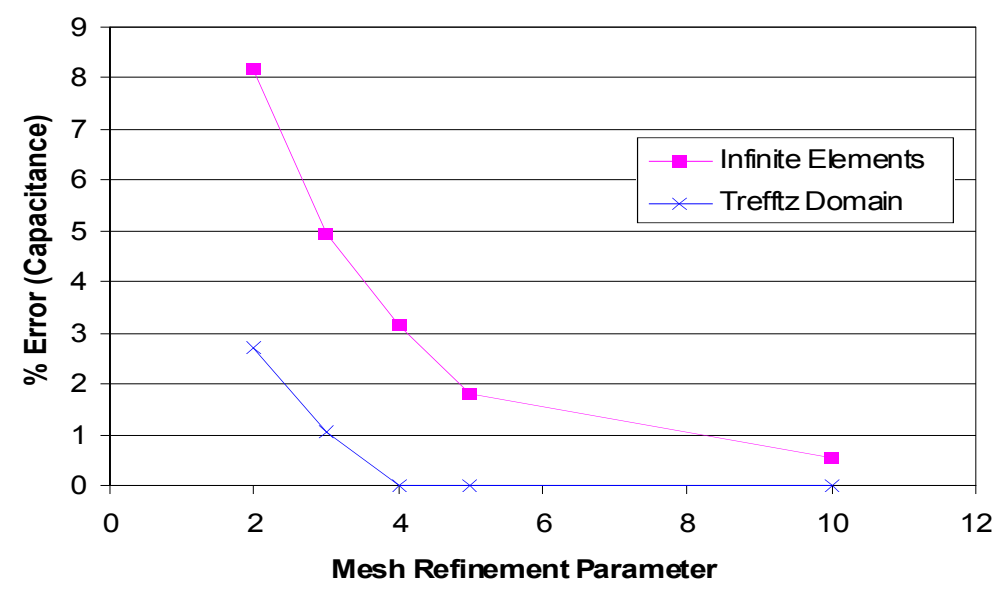

Figure 5: Trefftz and infinite elements convergence 